\title{
Promoted ABA Hydroxylation by Capsicum annuum CYP707As Overexpression Suppresses Pollen Maturation in Nicotiana tabacum
}

\author{
Hyun Min Kim, Se Hee Park, Sang Hoon Ma, Seo Young Park, Chul-Ho Yun, \\ Geupil Jang* and Young Hee Joung* \\ School of Biological Sciences and Technology, Chonnam National University, Gwangju, South Korea
}

\section{OPEN ACCESS}

Edited by:

Dianella G. Howarth,

St. John's University, United States

Reviewed by:

Jian Wu,

China Agricultural University, China

Chris Helliwell,

Commonwealth Scientific and Industrial Research Organisation

(CSIRO), Australia

*Correspondence:

Geupil Jang

yk3@jnu.ac.kr

Young Hee Joung

yhjoung@jnu.ac.kr

Specialty section:

This article was submitted to Plant Development and EvoDevo,

a section of the journal

Frontiers in Plant Science

Received: 19 July 2020 Accepted: 16 November 2020 Published: 08 December 2020

Citation:

Kim HM, Park SH, Ma SH,

Park SY, Yun $C H$, Jang $G$ and Joung YH (2020) Promoted ABA Hydroxylation by Capsicum annuum

CYP707As Overexpression

Suppresses Pollen Maturation

in Nicotiana tabacum

Front. Plant Sci. 11:583767.

doi: 10.3389/fpls.2020.583767
Abscisic acid (ABA) is a key signaling molecule that mediates plant response to stress. Increasing evidence indicates that $A B A$ also regulates many aspects of plant development, such as seed germination, leaf development, and ripening. ABA metabolism, including ABA biosynthesis and degradation, is an essential aspect of ABA response in plants. In this study, we identified four cytochrome P450 genes (CaCYP707A1, 2, 3, and 4) that mediate ABA hydroxylation, which is required for ABA degradation in Capsicum annuum. We observed that CaCYP707A-mediated ABA hydroxylation promotes ABA degradation, leading to low levels of $A B A$ and a dehydration phenotype in 35S:CaCYP707A plants. Importantly, seed formation was strongly inhibited in 35S:CaCYP707A plants, and a cross-pollination test suggested that the defect in seed formation is caused by improper pollen development. Phenotypic analysis showed that pollen maturation is suppressed in 35S:CaCYP707A1 plants. Consequently, most 35S:CaCYP707A1 pollen grains degenerated, unlike nontransgenic (NT) pollen, which developed into mature pollen grains. Together our results indicate that CaCYP707A mediates ABA hydroxylation and thereby influences pollen development, helping to elucidate the mechanism underlying ABA-regulated pollen development.

Keywords: ABA hydroxylation, CaCYP707A, Capsicum annuum, cytochrome P450, pollen maturation, pollen viability, seed formation

\section{INTRODUCTION}

Plant hormones regulate plant physiology and growth by governing endogenous developmental programs and plant responses to environmental signals. Abscisic acid (ABA) is an essential hormone that mediates plant defense against environmental stresses (Sah et al., 2016). For example, plants with reduced ABA response show reduced tolerance to abiotic stresses (drought, salinity, and oxidative stresses), whereas plants with enhanced $A B A$ response exhibit improved tolerance (Verslues and Bray, 2006; Dong et al., 2011; Vishwakarma et al., 2017). In addition, increasing evidence shows that $\mathrm{ABA}$ is deeply involved in aspects of plant development such as germination, 
stomate formation, and development of roots, leaves, and fruit (Nakashima and Yamaguchi-Shinozaki, 2013; Tanaka et al., 2013; Leng et al., 2014; Chater et al., 2015; Steinbrecher and LeubnerMetzger, 2017; Yoshida et al., 2019).

The ABA signaling pathway comprises several signaling components, including PYRABACTIN RESISTANCE 1 (PYR1)/PYR-LIKE (PYL)/REGULATORY COMPONENT OF ABA RECEPTOR (RCAR), PROTEIN PHOSPHATASE TYPE 2C (PP2C), and SNF1-RELATED PROTEIN KINASE TYPE 2 (SnRK2) (Ma et al., 2009; Park et al., 2009; Umezawa et al., 2009; Vlad et al., 2010). Mutant plants with defects in expression of PYL and SnRK2 genes show suppressed ABA responses (Fujii et al., 2011; Gonzalez-Guzman et al., 2012), indicating that $\mathrm{ABA}$ signaling is critical for $\mathrm{ABA}$ responses in plants. Briefly, ABA signaling is initiated by perception of ABA by the PYR1/PYL/RCAR family of signaling components ( $\mathrm{Ma}$ et al., 2009; Park et al., 2009). PYR-ABA interaction suppresses the activity of $\mathrm{PP} 2 \mathrm{C}$ proteins, which are responsible for the repression of downstream kinases such as SnRK2s, which themselves are positive regulators of ABA signaling (Cutler et al., 2010; Antoni et al., 2012; Soon et al., 2012). The kinase activities of SnRK2s activate ABA-RESPONSIVE ELEMENT BINDING FACTORS, leading to expression of ABA-responsive genes and promoting the ABA response (Furihata et al., 2006; Finkelstein, 2013; Ng et al., 2014).

The $\mathrm{ABA}$ response is also regulated by $\mathrm{ABA}$ metabolism, which includes biosynthesis, conjugation, and degradation. ABA biosynthesis involves the production of the ABA precursor xanthoxin in plastids and the conversion of xanthoxin to ABA in the cytoplasm (Seo and Koshiba, 2002; Seiler et al., 2011). Xanthoxin production, carried out by 9-cis-epoxycarotenoid dioxygenase (NCED), is a key step in ABA biosynthesis (Iuchi et al., 2001; Schwartz et al., 2003; Tan et al., 2003). Mutant plants that lack NCED activity show suppressed ABA accumulation and responses, whereas plants that overexpress NCED exhibit enhanced ABA responses (Tan et al., 1997; Burbidge et al., 1999; Iuchi et al., 2001; Lefebvre et al., 2006; Rodrigo et al., 2006; Zhang et al., 2009; Sun et al., 2012; Priya and Siva, 2015). Free ABA can be further metabolized by UDP glucosyltransferase into an ABAglucose ester through conjugation with glucose (Dietz et al., 2000; Han et al., 2019).

Degradation is another key process controlling cellular ABA levels. Although ABA-glucose ester conjugation is involved in ABA degradation, it has been suggested that the main process by which ABA is degraded is ABA hydroxylation, which is mediated by several cytochrome P450 (CYP) monooxygenases (CYP707As) (Krochko et al., 1998; Dong and Hwang, 2014; Han et al., 2019). The activity of CYP707As produces $8^{\prime}$ hydroxy ABA, which spontaneously converts into phaseic acid (PA) and is further metabolized into dihydrophaseic acid by PA reductase (Kushiro et al., 2004; Saito et al., 2004; Nambara and Marion-Poll, 2005; Rodriguez, 2016; Weng et al., 2016). CYP707As with ABA 8'-hydroxylase activity were conserved during land plant evolution (Zheng et al., 2012), and there are three CYP707As in rice, four in Arabidopsis, and seven in papaya (Kushiro et al., 2004; Saika et al., 2007; Nelson et al., 2008). CYP707A genes show tissue-specific expression patterns that change with the developmental stage (Okamoto et al., 2006; Nitsch et al., 2009; Wang et al., 2013; Brun et al., 2019). In addition, similar to other genes involved in ABA signaling and biosynthesis, expression levels of CYP707As are regulated by abiotic stresses such as drought, salinity, and oxidative stress (Yang and Zeevaart, 2006; Okamoto et al., 2009; Ren et al., 2010; Zheng et al., 2012; Wang et al., 2013; Ji et al., 2014). Furthermore, knockout or knockdown of CYP707As promotes seed dormancy and fruit ripening by increasing ABA levels (Kushiro et al., 2004; Okamoto et al., 2009; Ji et al., 2014), and inactivation of CYP707As with a chemical inhibitor mimics the effect of CYP707A mutation (Suttle et al., 2012), supporting that CYP707As play an essential role in regulation of $\mathrm{ABA}$ metabolism and response.

Hot pepper (Capsicum annuum) is a widely cultivated crop around the world (Pathirana, 2013), and the fruit productivity and quality are sensitive to environmental stresses (GuangCheng et al., 2010; Navarro et al., 2010; Unlukara et al., 2013). Because of this sensitivity, the role of ABA in stress responses in $C$. annuum has been widely studied (Lee and Choi, 2013; Guan et al., 2018; Hou et al., 2018; Jin et al., 2019; Ruggiero et al., 2019; Xiao et al., 2020). In this study, we attempted to isolate CYPs mediating ABA hydroxylation in C. annuum and identified four: CaCYP707A1, 2, 3, and 4. Enzymatic assays showed that the CYP707As have ABA hydroxylation activity, and expression analysis indicated that CaCYP707A transcription is regulated by abiotic stresses such as drought. In addition, transgenic tobacco overexpressing CaCYP707A genes (35S:CaCYP707As) exhibited a dehydration phenotype under normal growth conditions, unlike nontransgenic (NT) control plants, suggesting that CaCYP707A genes are responsible for ABA metabolism and responses. Moreover, overexpression of CaCYP707As suppressed seed formation, and analysis of pollen viability and ultrastructure showed that the suppression of seed formation was caused by defects in pollen maturation. These findings suggest that CaCYP707As-mediated ABA hydroxylation is involved in pollen maturation and seed formation.

\section{MATERIALS AND METHODS}

\section{Plant Materials and Growth Conditions}

Nicotiana tabacum cv. Xanthi-nc was used to generate 35S:CaCYP707A and NT control plants. Capsicum annuum L. cv. Bukang was used for isolation of CaCYP707As and for characterization of $\mathrm{CaCYP707A}$ expression in response to drought. All plants were grown at $22 \pm 1{ }^{\circ} \mathrm{C}$ with a controlled light $\left(120 \mu \mathrm{mol} \mathrm{m}^{-2} \mathrm{~s}^{-1}\right)$ cycle (16 h light/ $8 \mathrm{~h}$ dark).

\section{Isolation of CYP707As and Multiple Alignment}

To isolate CYP707As, total RNA extraction and cDNA biosynthesis from $C$. annuum leaves and flowers were carried out as described previously (Kim et al., 2019). The full-length CaCYP707A cDNAs were amplified by 
PCR from the C. annuum cDNA and cloned into pGEM$\mathrm{T}$ Easy Vectors (Promega, United States). The inserted sites were sequenced and aligned with their homologs from Arabidopsis and tomato using the PRALINE toolbox (Simossis and Heringa, 2005). CaCYP707A protein sequences were deduced from the nucleic acid sequences and uploaded to GenBank with the following accession numbers: CaCYP707A1 (MT198680), CaCYP707A2 (JQ828939), CaCYP707A3 (MT198681), and CaCYP707A4 (MT198682). Primer information for isolation of $C a C Y P 707 A$ genes is listed in Supplementary Table 1.

\section{Subcellular Localization of CaCYP707As}

Subcellular localization analysis was carried out as previously described (Voinnet et al., 2003; Kim et al., 2016), with a slight modification. 35S:CaCYP707A-GFP plasmids were constructed by inserting full-length of $\mathrm{CaCYP707A} \mathrm{cDNAs}$ into a pBI121-GFP plasmid carrying the $35 \mathrm{~S}$ promoter and the GFP gene; these were inserted into tobacco leaves (Nicotiana benthamiana) by agro-infiltration. To verify the subcellular localization of CaCYP707A-GFP, endoplasmic reticulum (ER)-targeting mCherry protein (ER-mCherry) and plasma membrane (PM)-targeting mCherry protein (PMmCherry) plasmids were used (Nelson et al., 2007); these were co-introduced into tobacco leaves together with the 35S:CaCYP707A-GFP plasmids. After 5 days, GFP and mCherry fluorescence signals were observed with a laser-scanning confocal microscope (Leica TCS SP5, Germany). Primer information for construction of 35S:CaCYP707A-GFP is listed in Supplementary Table 1.

\section{Analysis of ABA Hydroxylation Activity of CaCYP707As}

Full-length $C a C Y P 707 A$ cDNAs were ligated to $\mathrm{pCW}$ vectors and introduced into the Escherichia coli strain Rosetta (DE3) pLysS. The expression and purification of CaCYP707As were conducted as previously described (Kim et al., 2008). The concentrations of isolated CaCYP707A proteins were determined using the co-difference spectra method (Omura and Sato, 1964). The isolated CaCYP707A proteins were incubated with substrate (0.1 mM ABA), reductase, and a NADPH-generating system (NGS) in 0.1 M KPi buffer ( $\mathrm{pH} 7.4$ ). As the reductase, CaCPR1, which was confirmed to have reductase activity in a previous study (Lee et al., 2014), was used. The NGS contained $10 \mathrm{mM}$ glucose-6-phosphate, $0.5 \mathrm{mM} \mathrm{NADP}{ }^{+}$, and $1 \mathrm{IU} / \mathrm{ml}$ glucose 6phosphate dehydrogenase (Spaans et al., 2015). The negative controls were conducted without NGS solution. After a 30min incubation at $27^{\circ} \mathrm{C}$, the reaction was terminated with $25 \mu \mathrm{l}$ of $1 \mathrm{~N} \mathrm{HCl}$. The reaction products were collected by addition of ethyl acetate (three times) and dried using a stream of nitrogen gas. Analysis of product formation was conducted using an HPLC system (Shimadzu, Japan) with a UV detector and a Gemini C18 column $(4.6 \mathrm{~mm} \times 150 \mathrm{~mm}$, $5 \mu \mathrm{m}$, Phenomenex). The mobile phase solution was $0.05 \%$ acetic acid and $45 \%$ methanol in water $(\mathrm{v} / \mathrm{v})$. The flow rate was $1 \mathrm{ml} / \mathrm{min}$, and detection was conducted at $254 \mathrm{~nm}$.
Primer information for insertion into $\mathrm{pCW}$ vectors is listed in Supplementary Table 1.

\section{Generation of 35S:CaCYP707A Transgenic Plants}

To construct 35S:CaCYP707A genes, full-length CaCYP707A cDNAs were inserted into the pBI121 binary vector with the $35 \mathrm{~S}$ promoter for overexpression of CaCYP707As. The recombinant plasmids were introduced into tobacco using Agrobacterium tumefaciens strain LBA4404-mediated transformation as described previously (Oh et al., 2005), with a slight modification. For transgenic plant generation, tobacco leaves ( $N$. tabacum cv. Xanthi-nc) were cut into small disks and incubated with A. tumefaciens for $10 \mathrm{~min}$, after which the explants were placed on co-culture medium. After 2 days, explants were transferred to shoot induction medium [Murashige and Skoog medium containing 3\% (w/v) sucrose, $1 \mu \mathrm{g} / \mathrm{ml}$ zeatin, $0.01 \mu \mathrm{g} / \mathrm{ml} 1$ naphthaleneacetic acid, $0.1 \mu \mathrm{g} / \mathrm{ml}$ gibberellic acid, $50 \mu \mathrm{g} / \mathrm{ml}$ kanamycin, and $250 \mu \mathrm{g} / \mathrm{ml}$ cefotaxime, $\mathrm{pH}$ 5.8] and then transferred to new medium every 2 weeks. After 6-8 weeks, the developing shoots were excised from the callus and transferred to rooting medium [Murashige and Skoog medium containing $1.5 \%(\mathrm{w} / \mathrm{v}$ ) sucrose, $2 \mu \mathrm{g} / \mathrm{ml} 1$-naphthaleneacetic acid, $50 \mu \mathrm{g} / \mathrm{ml}$ kanamycin, and $250 \mu \mathrm{g} / \mathrm{ml}$ cefotaxime, $\mathrm{pH}$ 5.8]. After the root induced, these regenerated plants were transferred to soil. Primer information for construction of 35S:CaCYP707A genes is listed in Supplementary Table 1.

\section{Phenotypic Analysis of Pollen Development}

For the analysis of pollen ultrastructure, anthers were collected from tobacco flowers at early and middle developmental stages and then were fixed and observed as described previously (Lee et al., 2017), with minor modifications. In brief, the anthers were fixed in $0.05 \mathrm{M}$ sodium cacodylate buffer $(\mathrm{pH} 7.2)$ containing $2 \%(\mathrm{v} / \mathrm{v})$ glutaraldehyde and $2 \%(\mathrm{w} / \mathrm{v})$ paraformaldehyde and postfixed with $1 \%(\mathrm{w} / \mathrm{v})$ osmium tetroxide (Sigma-Aldrich, United States) in the same buffer. After a series of ethanol dehydrations, each sample was embedded in LR White resin (Sigma-Aldrich, United States). For light-microscopic observation, semi-thin section samples were cut with a diamond knife at $2-\mu \mathrm{m}$ increments in an ultramicrotome (RMC MT-X, United States) and stained with $0.5 \%(\mathrm{w} / \mathrm{v})$ toluidine blue $\mathrm{O}$ (Sigma-Aldrich, United States). These samples were visualized using an Axio Lab A1 light microscope (Zeiss, Germany). For transmission electron microscopy (TEM), ultra-thin (80- to 100$\mathrm{nm}$ ) section samples were sliced using the instruments named above and collected on a nickel grid (carbon-film coated, 150 mesh). These samples were stained with $4 \%(\mathrm{w} / \mathrm{v})$ uranyl acetate and $0.4 \%(\mathrm{w} / \mathrm{v})$ lead citrate. Images of the ultra-thin section samples were obtained using a JEM-2100F microscope/camera (Jeol Ltd., Japan). For phenotypical analysis of pollen by scanning electron microscopy (SEM), pollen at the anthesis stage of flowering were collected with carbon sticky tape and coated with platinum. The images of pollen were captured with a JSM-IT300 microscope/camera (Jeol Ltd., Japan). 


\section{Pollen Viability Assay}

To test pollen viability, the pollen collected from flowers at the anthesis stage were incubated in staining solutions containing $12.5 \mu \mathrm{g} / \mathrm{ml}$ fluorescein diacetate (FDA) and $5 \mu \mathrm{g} / \mathrm{ml}$ propidium iodide (PI) for $5 \mathrm{~min}$. Fluorescent signals (green signals for FDA; red for PI) were observed by fluorescence microscopy (Leica DM LB2, Germany).

\section{Quantitative PCR Analysis}

Capsicum annuum leaves were detached from 5-week-old plants and then air-dried (drought-stressed) for $6 \mathrm{~h}$ in the dark condition. Total RNA was extracted from these samples, and cDNA was biosynthesized as described previously (Kim et al., 2019). To analyze changes of $C a C Y P 707 A$ expression in response to drought, quantitative PCR (qPCR) analysis was conducted with using a Rotor-Gene 6000 real-time amplification system (Corbett Research, Australia). The reaction mix consisted of cDNA, gene-specific primers, and QuantiTect SYBR Green Master Mix (Qiagen, Germany). All qPCR experiments were run three times in three biological replicates. The relative expression of the target gene for each repeated experiment was calculated by the $2^{-\Delta \Delta C t}$ method as described previously (Livak and Schmittgen, 2001). Primer information for qPCR is listed in Supplementary Table 1.

\section{Quantification of ABA Concentration}

Abscisic acid concentrations were measured as previously described (Han et al., 2012), with slight modifications. To analyze ABA concentrations in leaves and anthers, $100 \mathrm{mg}$ samples of leaves were collected from 6-week-old tobacco plants and $100 \mathrm{mg}$ of anthers were collected from the flowers at the anthesis stage, respectively. The collected samples were suspended in $1.5 \mathrm{ml}$ of ABA extraction solution [0.45 mM butylated hydroxytoluene and $2.5 \mathrm{mM}$ citric acid monohydrate in $80 \%(\mathrm{v} / \mathrm{v})$ methanol] and incubated overnight at $4^{\circ} \mathrm{C}$. Supernatants were collected and dried under vacuum conditions. Extracted ABA amounts were measured using an ABA ELISA quantitation kit (Agrisera, Sweden) according to the manufacturer's instructions.

\section{RESULTS}

\section{Identification of CYP707As in C. annuum}

Abscisic acid hydroxylation, which is involved in ABA degradation, regulates the ABA response in plants (Nambara et al., 2010; Ma et al., 2018). CYP707As mediate ABA hydroxylation (Krochko et al., 1998). Through bioinformatics approaches, we identified four CYP707A genes in the C. annuum genome, similar to the numbers in Arabidopsis and tomato (Kushiro et al., 2004; Nitsch et al., 2009). The four predicted C. annuum CaCYP707A proteins (CaCYP707A1, 2, 3, and 4) are highly conserved, as are those of Arabidopsis and tomato (Supplementary Figure 1). These findings suggest that the CaCYP707A genes encode CYP proteins that contribute to ABA hydroxylation. Because ABA is hydroxylated in ER by the activity of ER-localized CYP707As (Seo, 2014), we investigated subcellular localization of CaCYP707A1, 2, 3, and 4 by visualizing localization of GFP-fused CaCYP707A1, 2, 3, and 4 proteins. CaCYP707A1-GFP, CaCYP707A2GFP, and CaCYP707A3-GFP signals were detected in the ER, where they co-localized with an ER marker. These findings suggest that the CaCYP707A1, 2, and 3 genes encode CYPs that are responsible for ABA hydroxylation in the ER. By contrast, CaCYP707A4 localized to the plasma membrane (Figure 1).

\section{ABA Hydroxylation Activity of CaCYP707As}

We next investigated whether the CaCYP707As had ABA hydroxylation activity (Figure 2). Previous studies demonstrated that CYP707As hydroxylate ABA to $8^{\prime}$-hydroxy ABA only in the presence of NADPH. The $8^{\prime}$-hydroxy ABA is spontaneously isomerized into $\mathrm{PA}$, so that ABA hydroxylation by CYP707As results in production of $8^{\prime}$-hydroxy ABA or PA (Saito et al., 2004). When the CaCYP707A1 protein was incubated with ABA in the presence of NAPDH, 8'-hydroxy ABA and PA peaks were detected. By contrast, the peaks were not observed in NAPDHfree conditions in which CaCYP707As cannot act, indicating that CaCYP707A1 have the activity of ABA hydroxylation (Figure 2A). Similar to CaCYP707A1 proteins, CaCYP707A2, $\mathrm{CaCYP707A3}$, and CaCYP707A4 proteins gave rise to $8^{\prime}$ hydroxy $\mathrm{ABA}$ and $\mathrm{PA}$ peaks only in the presence of $\mathrm{NADPH}$, indicating that they also have ABA hydroxylation activity (Figures 2B-D). These findings indicate that the CaCYP707A genes encode CYPs responsible for ABA hydroxylation in $C$. annuum, and suggest that $C a C Y P 707 A$ is involved in ABA metabolism. This idea is partially supported by the result that transcriptional expression of $\mathrm{CaCYP707A}$ genes is regulated by abiotic stress such as dehydration conditions (Supplementary Figure 2).

\section{CaCYP707A Negatively Regulate Seed Formation}

To understand the function of $C a C Y P 707 A$ genes in plant development, we generated transgenic tobacco plants overexpressing CaCYP707A1 (35S:CaCYP707A1), CaCYP707A2 (35S:CaCYP707A2), СaCYP707A3 (35S:CaCYP707A3), and CaCYP707A4 (35S:CaCYP707A4). In the 35S:CaCYP707A transgenic plants, the ABA concentration was approximately 2-6 fold lower than that of NT grown in the same conditions for 6 weeks (Supplementary Figure 3). In addition, the transgenic plants exhibited a dehydration-like phenotype under normal growth conditions, unlike NT plants (Supplementary Figure 4). Because overexpression of $\mathrm{CaCYP707As}$ reduces ABA levels by promoting hydroxylation of ABA, a key hormone of stomatal closure, it was suggested that the dehydration-like phenotype of CaCYP707As-overexpressing plants might be caused by preventing ABA-induced stomatal closure.

Importantly, we observed that 35S:CaCYP707A plants showed defects in seed formation, although there was no obvious difference in floral structure between NT and 35S:CaCYP707A plants. The total number of seeds harvested 

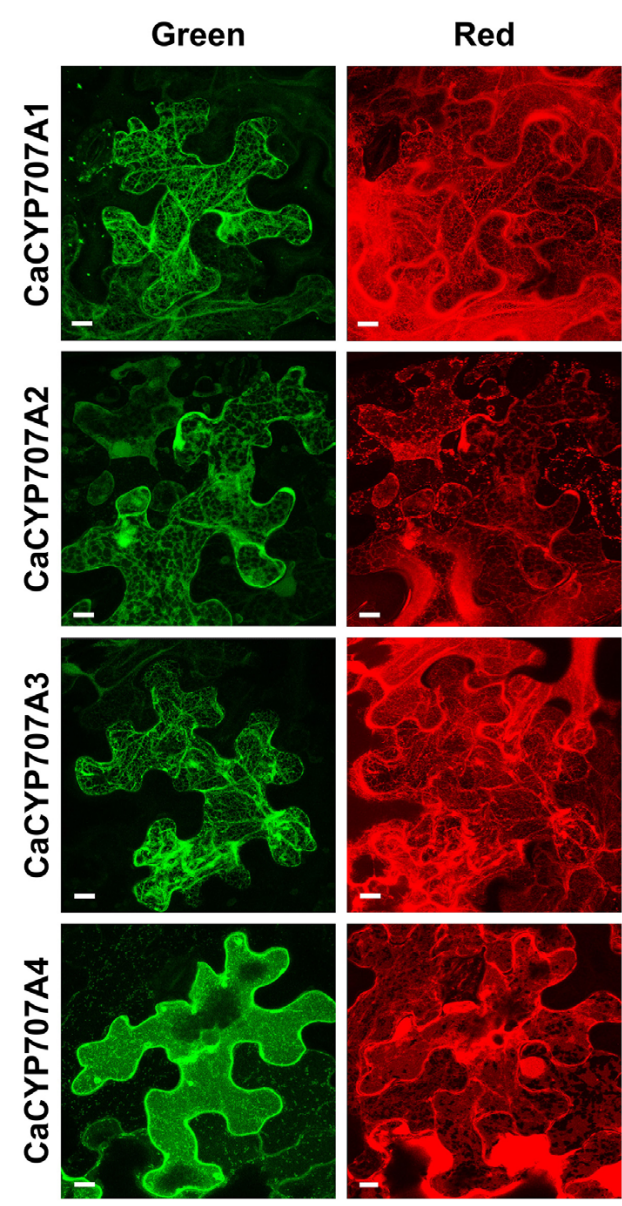

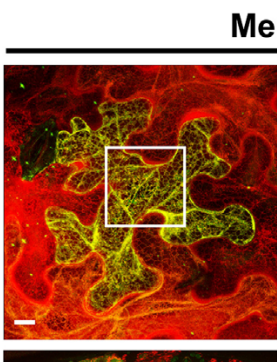

\section{Merge}
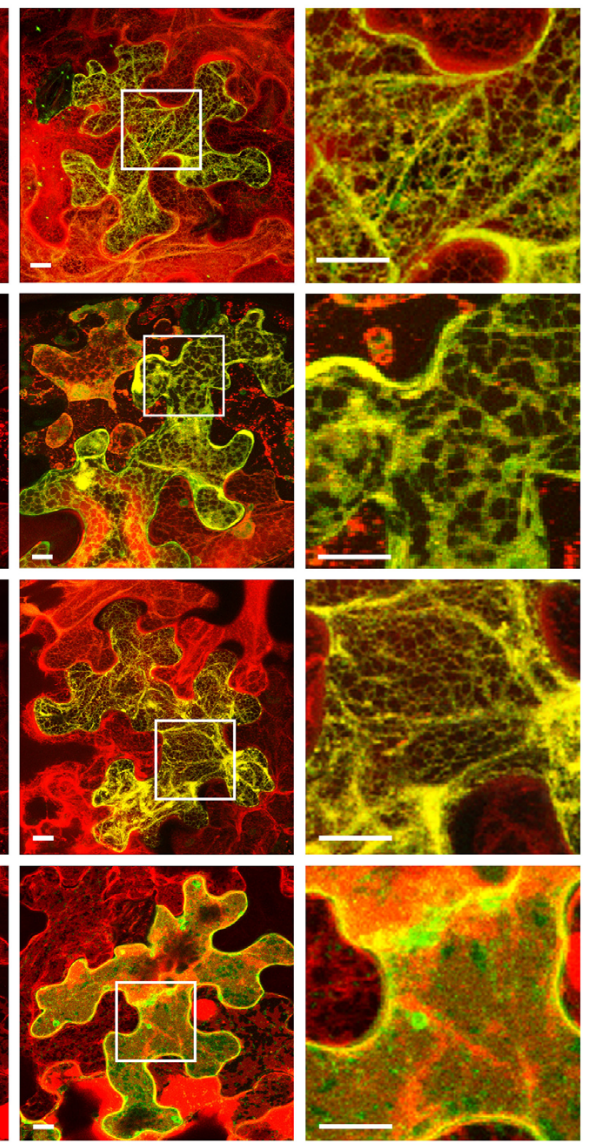

FIGURE 1 | Subcellular localization of CaCYP707As. Subcellular localization of CaCYP707A1, 2, 3, and 4 proteins fused to GFP was investigated by visualizing fluorescent signals. ER-specific localization of CaCYP707A1, 2, and 3 proteins and PM-specific localization of CaCYP707A4 was verified through co-localization of ER-targeting mCherry (ER-mCherry) and PM-targeting mCherry (PM-mCherry), respectively. CYP707A1, 2, 3, and 4 indicate tobacco transiently expressing 35S:CaCYP707A1-GFP, 2-GFP, 3-GFP, and 4-GFP. Green and red indicate fluorescence signals of GFP and mCherry, respectively. Scale bars = $10 \mu \mathrm{m}$. More than three individuals of the indicated plants were analyzed, and the experiments were performed three times with similar results.

from NT plants was approximately 25,000. However, total numbers of seeds collected from transgenic plants grown in the same conditions were much lower; 35S:CaCYP707A1 plants produced $\sim 800-1,400,35 S: C a C Y P 707 A 2$ plants produced 2,000-4,200, 35S:CaCYP707A3 plants produced 1,000-3,000, and 35S:CaCYP707A4 plants produced 1,000-8,000 (Figure 3). The transgenic plants had produced similar or slightly lower numbers of flowers compared to wild-type plants. Consequently, the ratios of seed number and flower number in the transgenic plants were low compared to that of NT plants. This result also implies that the lower number of seeds in the transgenic plants is caused by defects in fertilization but not by defects in flower development. To explore this, we performed crosspollination test. Seed formation in CaCYP707As-overexpressing plants was increased by cross-pollination using NT pollens. By contrast, seed formation in NT plants was decreased by crosspollination using the pollens of CaCYP707As-overexpressing plants (Figure 4). This finding indicated that the suppressed seed formation in 35S:CaCYP707A plants is caused by pollen defects in fertilization, suggesting that $C a C Y P 707 A$ are possibly involved in pollen development.

\section{CaCYP707A Genes Regulate Pollen Maturation}

To understand the role of $C a C Y P 707 A$ genes in pollen development, NT and 35S:CaCYP707A1 anthers were collected from flowers at two different stages, and the morphology of the pollen was visualized by semi-thin sectioning and toluidine blue staining (Figure 5). In the early stage NT flowers, all pollen grains displayed a shrunken shape, and the phenotype was almost identical to that of $35 \mathrm{~S}: \mathrm{CaCYP707A1}$ pollen grains. However, the morphologies of NT and 35S:CaCYP707A1 pollen in middlestage flowers differed. In the middle-stage NT flowers, most pollen grains were mature pollen grains (MPGs) with a spherical shape and only a few pollen grains were degenerated pollen grains (DPGs) with a shrunken shape (Xu et al., 2017; Zhu et al., 2017). By contrast, in middle-stage 35S:CaCYP707A1 flowers, 


\section{+ NGS}

A

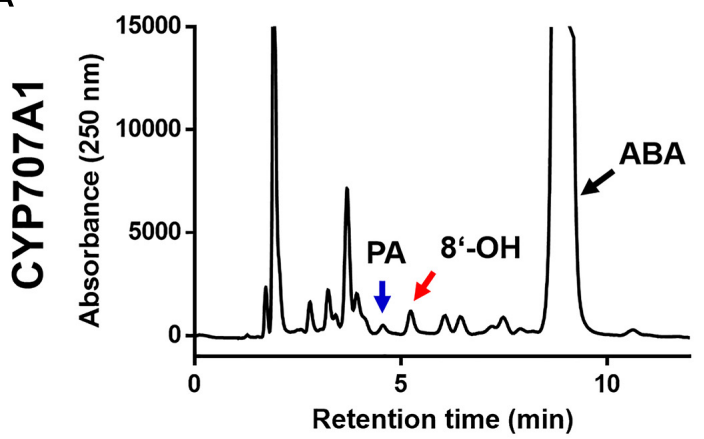

B

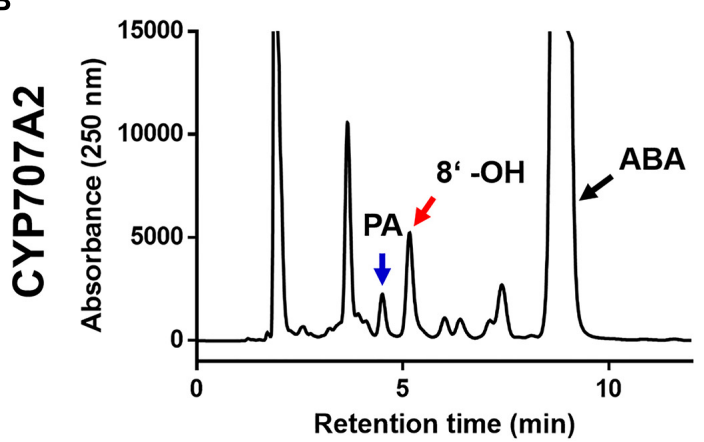

C

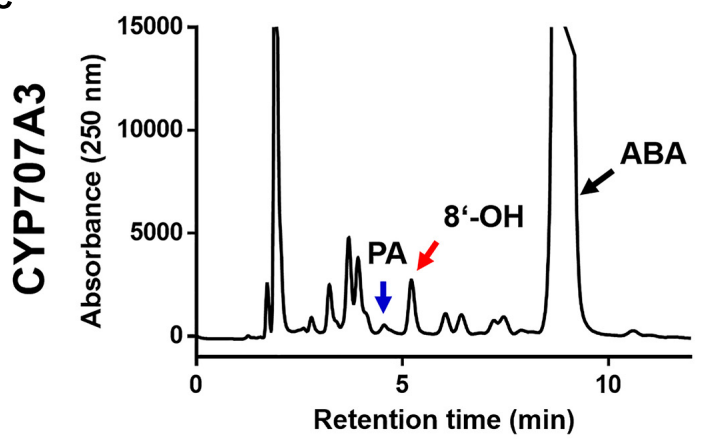

D

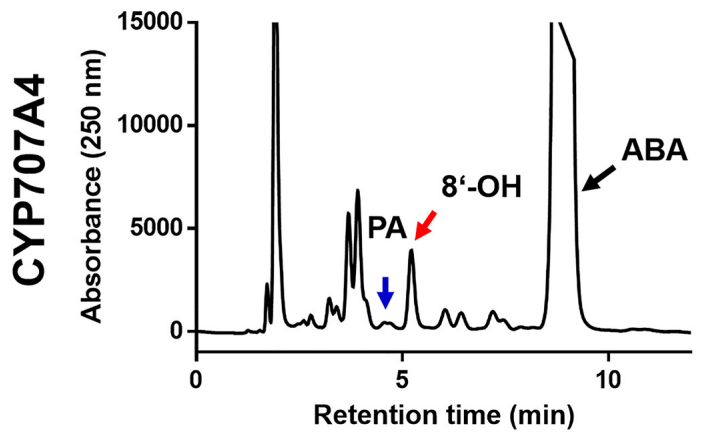

- NGS
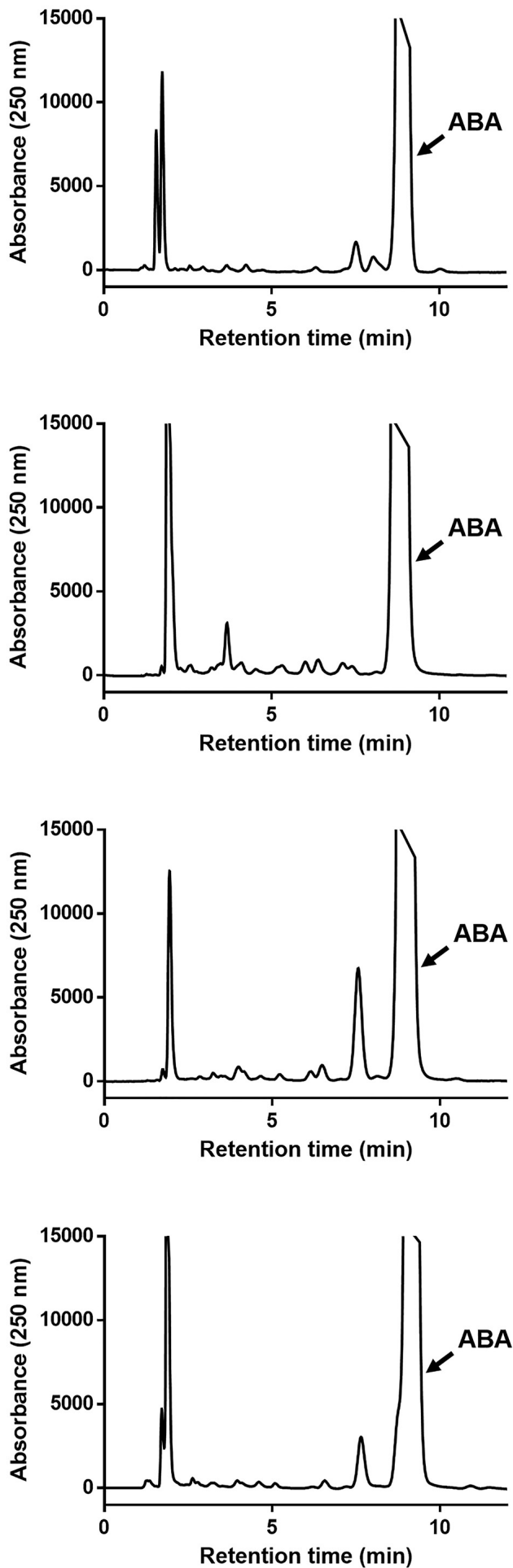

FIGURE 2 | ABA hydroxylation activity of CaCYP707As. Characterization of CaCYP707A1 (A), CaCYP707A2 (B), CaCYP707A3 (C), and CaCYP707A4 (D) function in ABA hydroxylation. CYP707A1, 2, 3, and 4 proteins expressed in E. coli were incubated with ABA in the presence (left; +NGS) or absence (right; -NGS) of a NADPH-generating system, and the catabolites were analyzed by HPLC. The peaks marked by red and blue arrows are $8^{\prime}$-hydroxy ABA ( $\left.8^{\prime}-\mathrm{OH}\right)$ and phaseic acid (PA), respectively, whereas the peaks marked by black arrows are abscisic acid (ABA). The experiments were performed three times with similar results. 

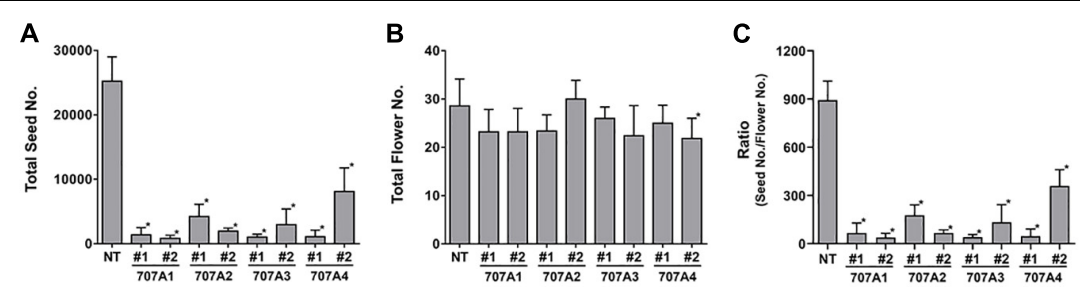

FIGURE 3 | Overexpression of CaCYP707As suppresses seed formation. (A) Quantification of total seed number harvested from NT and 35S:CaCYP707A plants grown in the same conditions $(n>5)$. 707A1, 2, 3, and 4 indicate 35S:CaCYP707A1, 2, 3, and 4 transgenic plants, respectively. \#1 and \#2 are two individual lines of the indicated plants. (B) Quantification of total numbers of flowers formed in NT and 35S:CaCYP707As plants ( $n>5)$. (C) Ratio of total seed number to the number of flowers. Error bars are S.D. Asterisks indicate significant differences between the transgenic plants and NT controls (Student $t$-test $p<0.01$ ). The experiments were performed three times with similar results.
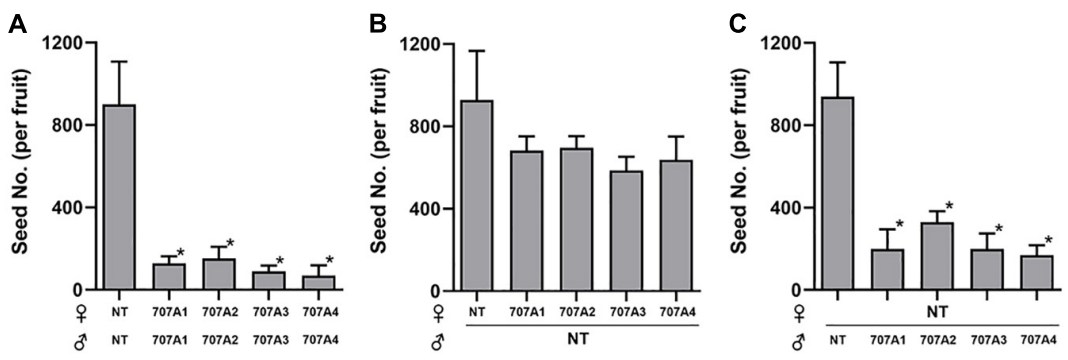

FIGURE 4 | Cross-pollination rescues defective seed formation in CaCYP707A-overexpressing plants. (A) The number of seeds were quantified in the NT and transgenic fruits generated by self-pollination $(n>5)$. (B) The number of seeds were quantified in the 35S:CaCYP707As transgenic fruits generated by cross-pollination using NT pollens $(n>5)$. (C) The number of seeds were quantified in the NT fruits generated by cross-pollination using the pollens of 35S:CaCYP707As transgenic plants $(n>15)$. NT, 707A1, 2, 3, and 4 indicate non-transgenic, 35S:CaCYP707A1, 2, 3, and 4, respectively. Error bars are S.D. Asterisks indicate significant differences between NT control and the indicated samples (Student $t$-test, $p<0.01$ ). Experiment $(\mathbf{A}, \mathbf{B})$ were performed three times with similar results.

most pollen grains were DPGs, and a few pollen grains showed an intermediate phenotype between those of DPGs and MPGs. These findings indicate that 35S:CaCYP707A1 transgenic plants have defects in pollen maturation, suggesting that overexpression of CaCYP707A1 negatively regulates maturation of pollen.

To further explore their maturation, we examined the ultrastructure of 35S:CaCYP707A1 pollen by TEM (Figure 6). Similar to the results from light microscopy, we observed morphological differences between NT and 35S:CaCYP707A1 pollen only in the middle-stage flowers with mature pollen grains, but not in early stage flowers with immature pollen grains. Notably, TEM analysis showed that ER development, which is tightly linked to pollen maturation (Kim et al., 2011), was different between NT and 35S:CaCYP707A1 pollen in middle-stage flowers. In middle-stage flowers, we observed welldeveloped ER only in NT pollen, but not in 35S:CaCYP707A1 pollen. However, this difference in ER formation was not observed between NT and 35S:CaCYP707A1 pollen in early stage flowers (Figure 6). These findings support the conclusion that overexpression of $C a C Y P 707 A 1$ suppresses pollen maturation.

\section{CaCYP707As Negatively Regulate Pollen Viability}

To further test the hypothesis that CaCYP707A1 negatively regulates maturation of pollen, we analyzed the morphologies of the NT and CaCYP707A1-overexpressing plant pollen at the anthesis stage using SEM. In NT plants, most pollen grains had similar size and shape. The 35S:CaCYP707A1 flowers formed a few pollen grains whose phenotype (size and shape) was similar to that of the NT pollen; however, most pollen in the transgenic flowers was smaller and more irregular compared to the pollen of NT flowers (Figure 7). This SEM result supports the TEM result showing that maturation of pollen is defective in 35S:CaCYP707A1 plants. In addition, the result showing that ABA concentration in the anthers of $35 \mathrm{~S}: \mathrm{CaCYP707A1}$ plants is much lower than that of NT plants (Supplementary Figure 5) suggests that $\mathrm{CaCYP707A}$-promoted ABA hydroxylation inhibits pollen maturation. Similar to $C a C Y P 707 A 1$, overexpression of CaCYP707A2, CaCYP707A3 or CaCYP707A4 also suppressed pollen maturation (Supplementary Figure 6), further supporting the function of $\mathrm{CaCYP707As}$ in pollen development.

To address how the defect in pollen maturation leads to the inhibited seed formation in 35S:CaCYP707As plants, we tested pollen viability in NT and 35S:CaCYP707A1 plants. To do this, we stained pollens at the anthesis stage with FDA and PI, and monitored the fluorescent signals of FDA and PI to visualize viability (Figure 8). In this assay, viable pollen exhibit green fluorescence signals upon FDA staining, whereas non-viable pollen display red fluorescence signals upon PI staining. In NT plants, most pollen displayed green fluorescence signals upon FDA staining. In contrast to the NT 


\section{Magnification}

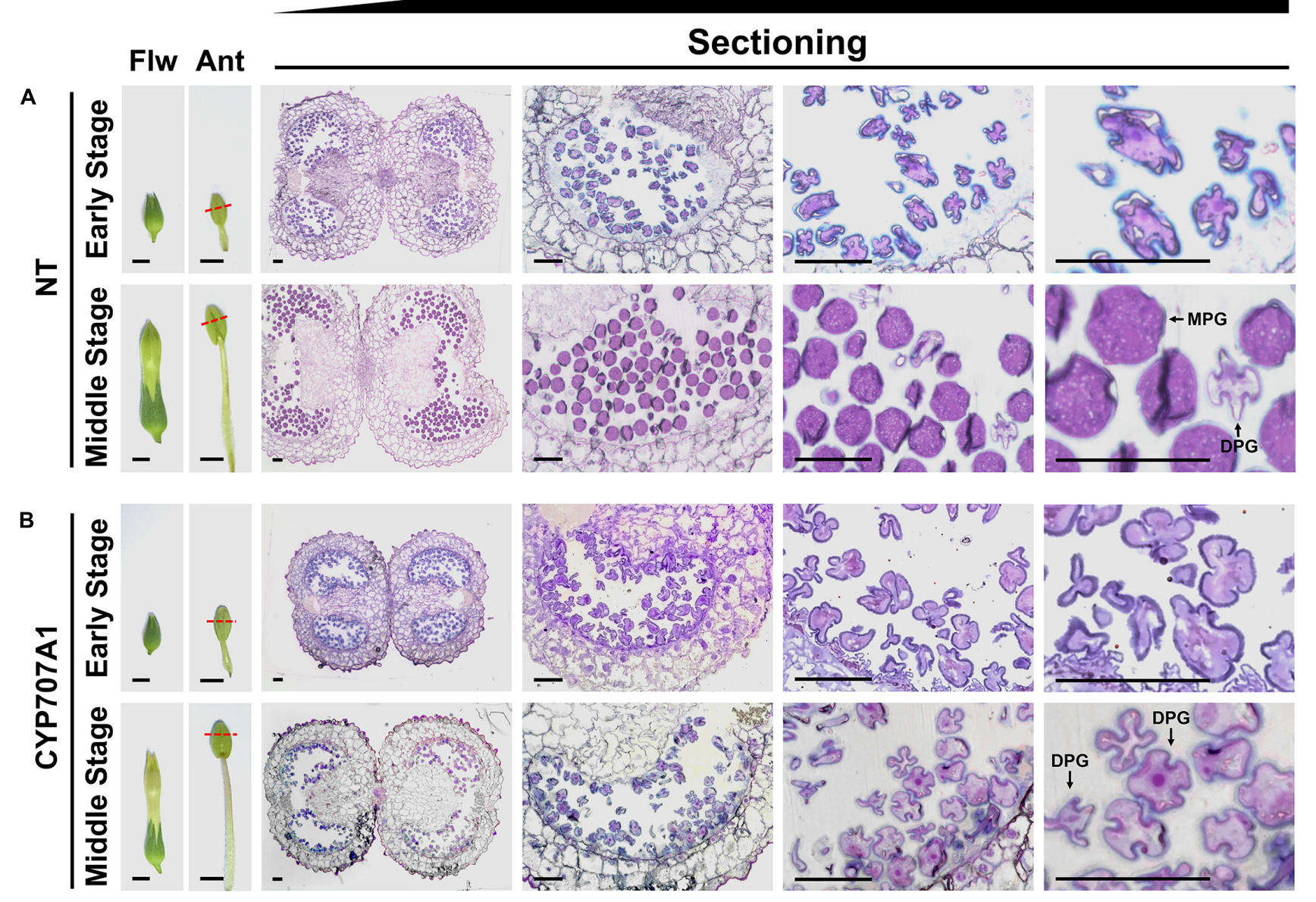

FIGURE 5 | Overexpression of CaCYP707A1 affects pollen maturation. (Left) Images of flowers (Flw) and anthers (Ant), and (right) semi-thin section images showing pollen development in early stage and middle-stage flowers of NT (A) and 35S:CaCYP707A1 \#1 (CYP707A1) plants (B) grown in the same conditions. The slices were stained with toluidine blue $\mathrm{O}$, and the images were captured by light microscope. Red dashed lines marked the longitudinal positions from which the slices were obtained. Ant, anther; DPG, degenerated pollen grain; Flw, flower; MPG, mature pollen grain. Scale bars = $0.5 \mathrm{~cm}$ in flowers, $0.2 \mathrm{~cm}$ in anthers, and $50 \mu \mathrm{m}$ in sectioning images. More than three individuals of the indicated plants were analyzed, and the experiments were performed two times with similar results.

plants, most pollen from 35S:CaCYP707A1 plants showed red fluorescence signals upon PI staining, whereas only a small portion of the pollen exhibited green fluorescence signals (Figures 8A,B). We obtained nearly identical results for the pollen viability of plants expressing CaCYP707A2, 3, and 4 . Most pollen in 35S:CaCYP707A2, 3, and 4 plants displayed red signals corresponding to PI, and only a few pollen exhibited green signals corresponding to FDA (Figures 8C-E). When we determined the ratio of the number of pollen with FDA signals to the number of pollen with PI signals, there was an obvious difference in pollen viability (Figure $\mathbf{8 F}$ ). The ratios of the transgenic plants were much lower than those of the NT controls. This finding indicates that CaCYP707Apromoted ABA hydroxylation negatively regulate pollen viability, explaining why seed formation was inhibited in 35S:CaCYP707A plants.

\section{DISCUSSION}

The plant CYPs are the third largest gene family in higher plants (Nelson and Werck-Reichhart, 2011). Unlike bacterial CYPs, most plant CYPs with transmembrane domains are mainly located at the ER, and some are predicted to be located at the membranes of other organelles such as PM and plastids (Schuler et al., 2006; Bak et al., 2011; Yin-Ping et al., 2019). CYPs act as monooxygenases, and their activity is regulated by NADPH and NADPH-cytochrome P450 reductase (CPR), which provide electrons to CYPs (Jensen and Moller, 2010; Bak et al., 2011). In plants, CYPs are involved in plant growth and physiology, mediating a variety of metabolic processes, including metabolism of hormones such as auxin, cytokinin, and ABA (Kim and Tsukaya, 2002; Mizutani, 2012). In this study, we identified four CYP707As (CaCYP707A1, 2, 3, and 4) in C. annuum and 


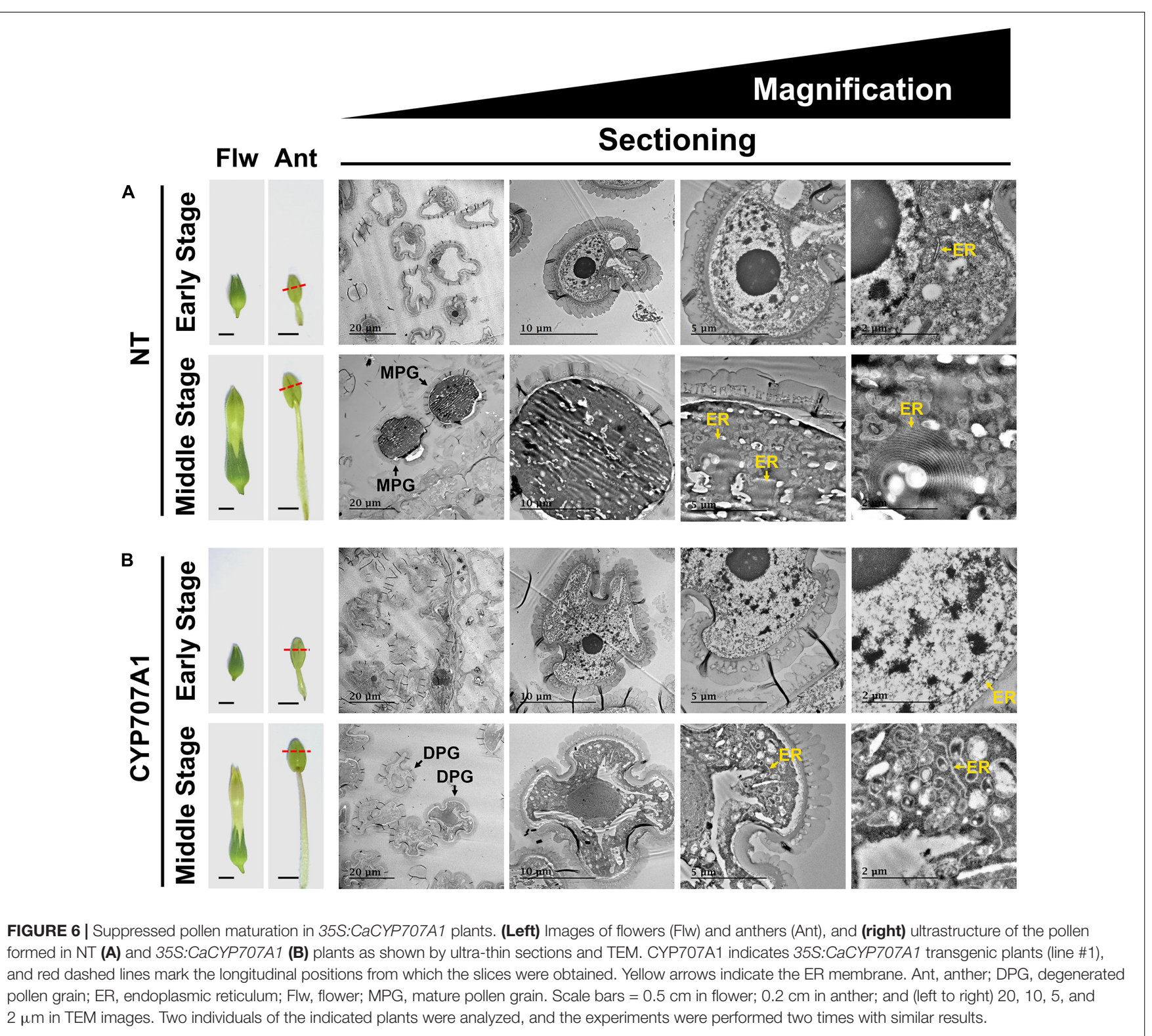

characterized their function in ABA degradation. Production of $8^{\prime}$-hydroxy ABA is a key step in ABA degradation (Saito et al., 2004), and our enzymatic assay showed that the CaCYP707As mediate production of 8 -hydroxy $\mathrm{ABA}$ from $\mathrm{ABA}$ only in the presence of NADPH. In addition, overexpression of $\mathrm{CaCYP707A}$ genes reduced $\mathrm{ABA}$ levels and induced a dehydration phenotype, indicating that the CaCYP707As mediate ABA degradation by controlling hydroxylation of ABA.

In this study, we observed that seed formation is suppressed by overexpression of $\mathrm{CaCYP707A}$ genes. The total numbers of seeds formed in 35S:CaCYP707A1 plants were $\sim 20$-fold lower than those of NT plants, and the defective phenotype was also

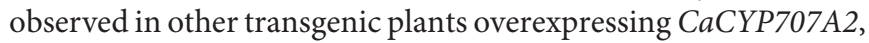
3 , and 4. This indicates that CaCYP707A1-mediated ABA degradation regulates seed formation. Previous studies using ABA biosynthesis mutants support this finding. ABA-deficient mutant aba2-1 shows suppressed ABA response and forms fewer seeds per silique in Arabidopsis (Sharma and Verslues, 2010; Cheng et al., 2014), and a knockdown mutant of NCED, which is responsible for ABA biosynthesis, also exhibits lower levels of ABA than NT control plants, leading to fewer seeds per fruit (Sun et al., 2012). Together, these findings suggest that CaCYP707Amediated degradation of ABA induces defects in seed formation, and the cross-pollination result showing that NT pollen rescued the defective seed phenotype in 35S:CaCYP707A plants further indicates that the defective seed phenotype is due to defects in pollen development in CaCYP707A-overexpressing plants.

A recent study by Dai et al. (2018) using NCED suggested that $\mathrm{ABA}$ is involved in pollen development in tomato. In that study, the authors showed that RNAi gene silencing of NCED reduced the ABA levels in the RNAi transgenic tomato and suppressed pollen development. Interestingly, overexpression of NCED also 


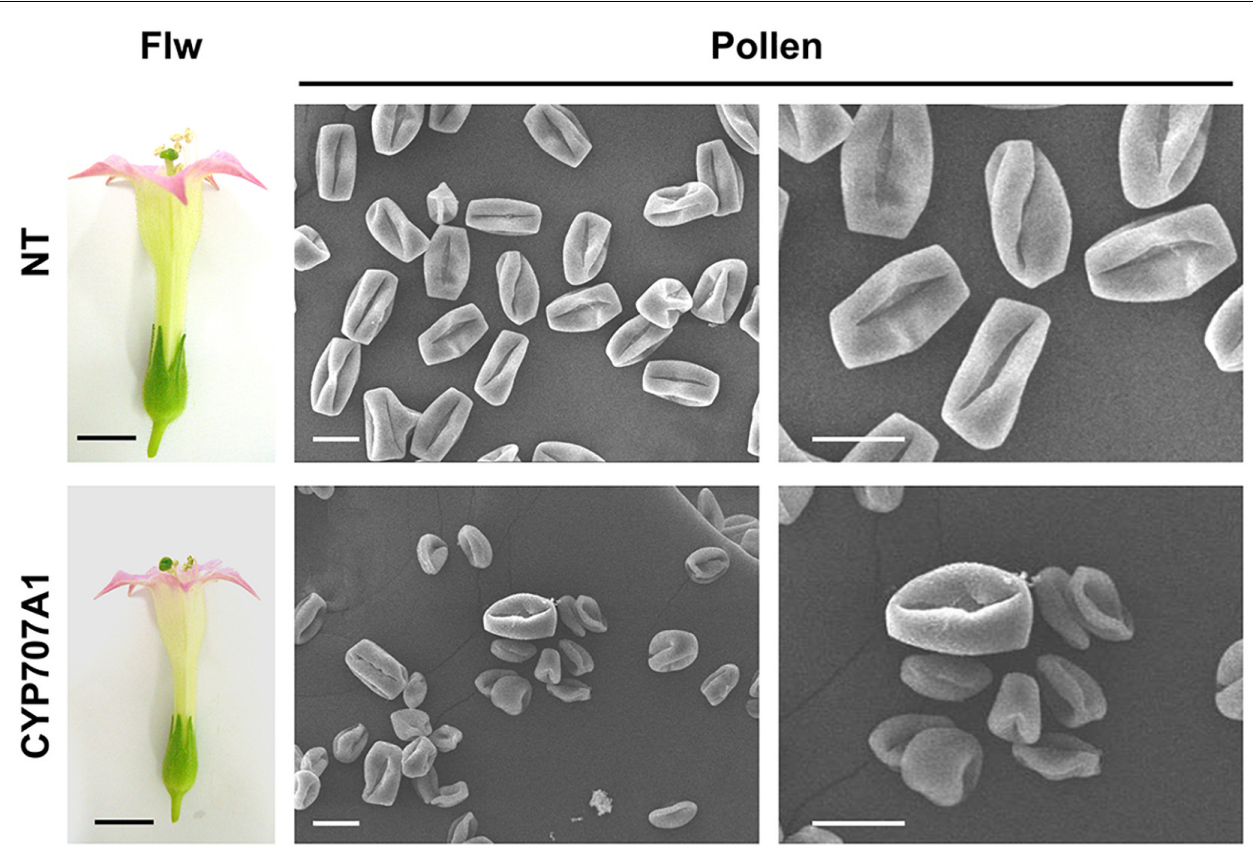

FIGURE 7 | Overexpression of CaCYP707A1 promotes formation of immature pollen. Pollen maturation was investigated by SEM. Pollen of NT and 35S:CaCYP707A1 (line \# 1) were collected from the anthesis stage of flowers (left), and morphological patterns of the pollen were captured by SEM (right). Flw and CYP707A1 indicate flowers and 35S:CaCYP707A1 plants, respectively. Scale bars $=1 \mathrm{~cm}$ in flowers and $20 \mu \mathrm{m}$ in SEM images. Two individuals of the indicated plants were analyzed, and the experiments were performed two times with similar results.

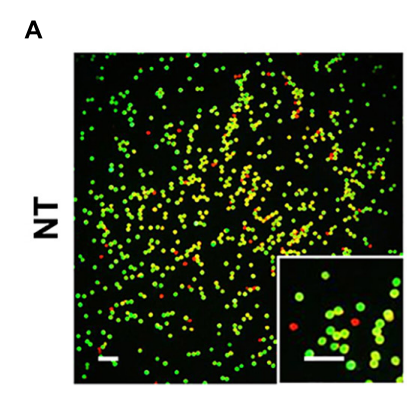

D

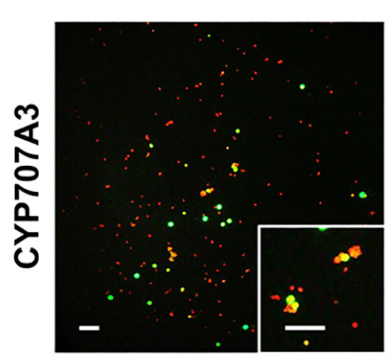

B

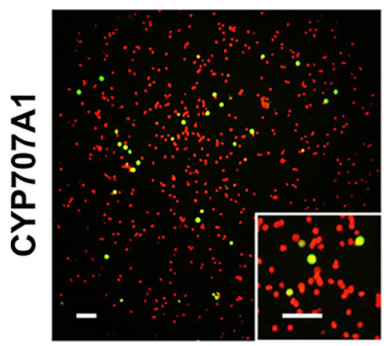

E

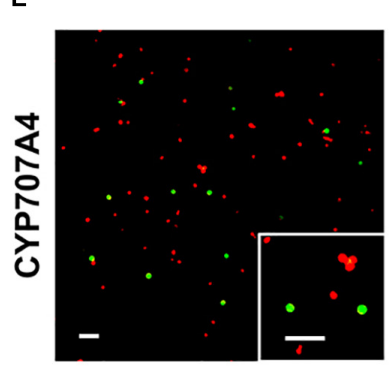

C

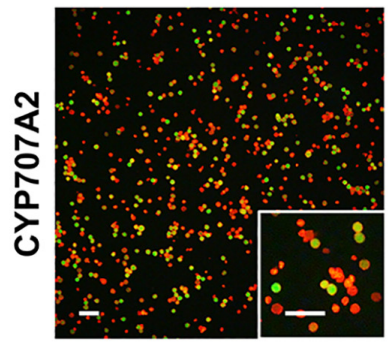

F

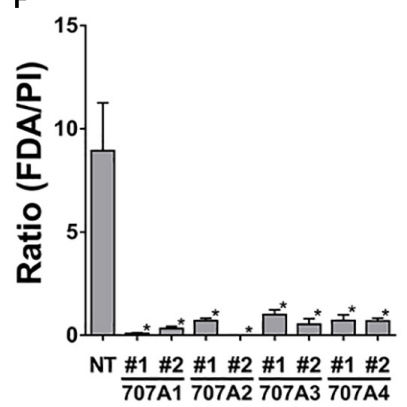

FIGURE 8 | Suppressed pollen viability in 35S:CaCYP707A plants. Pollen viability was visualized by FDA and PI staining. Pollen collected from the flowers (anthesis stage) of NT and 35S:CaCYP707A transgenic plants were stained with a mixture of FDA and PI for 5 min, and then fluorescent signals were observed by fluorescence microscopy [(A), NT; (B), CaCYP707A1 \#1; (C), CaCYP707A2 \#1; (D), CaCYP707A3 \#1; and (E), CaCYP707A4 \#1]. More than three individuals of the indicated plants were analyzed with similar results. (F) Quantification of the ratio of FDA (green) to PI (red) signals in the indicated plants ( $n>3$ ). The values are the means of three biological replicates, and error bars are S.D. \#1 and \#2 indicate individual lines of the indicated transgenic plants. Asterisks indicate significant differences between the transgenic plants and NT controls (Student $t$-test $p<0.01$ ). Scale bars $=100 \mu \mathrm{m}$. The experiments were performed at least three times with similar results. 
suppressed pollen development, although the overexpression increased ABA levels, suggesting that pollen development is sensitive to ABA homeostasis. A study by Saini and Aspinall (1982) showing that exogenous ABA affects pollen development partially supports this hypothesis. In our study, we traced pollen development with age, and found that maturation of immature pollen grains is suppressed in 35S:CaCYP707A1 transgenic plants. This indicated that overexpression of $C a C Y P 707 A$ genes suppresses pollen maturation, and ultra-thin sectioning and TEM results visualizing ER formation further supported this finding. Establishment of the intracellular membrane network during pollen maturation is characterized by ER development (Rodríguez-García et al., 1995; Piffanelli et al., 1998). Therefore, the TEM results that middle-stage 35S:CaCYP707A1 pollen had a poorly developed ER unlike the same developmental stage of NT pollens with a well-developed ER support the hypothesis that CaCYP707A negatively regulates pollen maturation. In addition, our SEM and pollen viability results show that the defect in pollen maturation affects pollen development and pollen viability. NT pollen grains at the anthesis stage had similar size and shape, and also were viable, whereas pollen grains of $35 \mathrm{~S}: \mathrm{CaCYP707A1}$ plants were irregular in shape and non-viable. Additionally, in response to exogenous ABA treatment, pollen maturation in NT and CaCYP707A1-overexpressing plants tended to be reduced and enhanced, respectively (Supplementary Figure 7). This indicates that overexpression of CaCYP707A1 suppressed by promoting ABA hydroxylation, and also suggests that ABA homeostasis is involved in pollen maturation. This finding is partially supported by a previous study by Dai et al. (2018), which shows that ABA homeostasis affects pollen development (Dai et al., 2018). Together with the results showing that CaCYP707A1 overexpression reduced ABA levels in anthers, these findings suggest that overexpression of CaCYP707As suppresses pollen maturation and seed formation by promoting ABA hydroxylation.

\section{DATA AVAILABILITY STATEMENT}

The datasets presented in this study can be found in online repositories. The names of the repository/repositories and accession number(s) can be found in the article/ Supplementary Material.

\section{REFERENCES}

Antoni, R., Gonzalez-Guzman, M., Rodriguez, L., Rodrigues, A., Pizzio, G. A., and Rodriguez, P. L. (2012). Selective inhibition of clade A phosphatases type 2C by PYR/PYL/RCAR abscisic acid receptors. Plant Physiol. 158, 970-980. doi: 10.1104/pp.111.188623

Bak, S., Beisson, F., Bishop, G., Hamberger, B., Hofer, R., Paquette, S., et al. (2011). Cytochromes p450. Arabidopsis Book 9:e0144.

Brun, G., Thoiron, S., Braem, L., Pouvreau, J. B., Montiel, G., Lechat, M. M., et al. (2019). CYP707As are effectors of karrikin and strigolactone signalling pathways in Arabidopsis thaliana and parasitic plants. Plant Cell Environ. 42, 2612-2626. doi: 10.1111/pce.13594

Burbidge, A., Grieve, T. M., Jackson, A., Thompson, A., Mccarty, D. R., and Taylor, I. B. (1999). Characterization of the ABA-deficient tomato mutant notabilis and

\section{AUTHOR CONTRIBUTIONS}

HMK and YHJ planned and designed all aspects of the research. HMK and CHY analyzed the data and provided technical guidance in enzyme assay experiments. HMK and SHP generated plant gene expression constructs. HMK, SHM, and SYP participated phenotypical analysis of plant. HMK, GJ, and YHJ performed the data interpretation and wrote the manuscript. All authors contributed to the article and approved the submitted version.

\section{FUNDING}

This work was supported by a grant from the New Breeding Technologies Development Program (Project No. PJ01487401) through the Rural Development Administration (RDA) and the Basic Research Lab Program (NRF-2018R1A4A1023882) through the National Research Foundation of Korea (NRF), South Korea. This study was financially supported by Chonnam National University (Grant No. 2020-1850).

\section{SUPPLEMENTARY MATERIAL}

The Supplementary Material for this article can be found online at: https://www.frontiersin.org/articles/10.3389/fpls.2020. 583767/full\#supplementary-material

\section{Supplementary Figure 1 | Multiple alignment of CaCYP707As amino acid sequence.}

Supplementary Figure 2 | CaCYP707As expression in response to drought.

Supplementary Figure 3 | ABA concentration of CaCYP707As transgenic plant leaves.

Supplementary Figure 4 | Dehydration phenotype of CaCYP707As transgenic plants.

Supplementary Figure 5 | ABA concentration of CaCYP707A1 transgenic plant anther.

Supplementary Figure 6 | Morphological patterns of CaCYP707A2, 3, and 4 transgenic plant pollen.

Supplementary Figure 7 | Pollen maturation of CaCYP707A1 transgenic plant under ABA treatment.

Supplementary Table 1 | A list of primer sequences used in this study.

its relationship with maize Vp14. Plant J. 17, 427-431. doi: 10.1046/j.1365313x.1999.00386.x

Chater, C., Peng, K., Movahedi, M., Dunn, J. A., Walker, H. J., Liang, Y. K., et al. (2015). Elevated $\mathrm{CO} 2$-induced responses in stomata require $\mathrm{ABA}$ and ABA Signaling. Curr. Biol. 25, 2709-2716. doi: 10.1016/j.cub.2015. 09.013

Cheng, Z. J., Zhao, X. Y., Shao, X. X., Wang, F., Zhou, C., Liu, Y. G., et al. (2014). Abscisic acid regulates early seed development in Arabidopsis by ABI5-Mediated Transcription of SHORT HYPOCOTYL UNDER BLUE1. Plant Cell 26, 1053-1068. doi: 10.1105/tpc.113. 121566

Cutler, S. R., Rodriguez, P. L., Finkelstein, R. R., and Abrams, S. R. (2010). Abscisic acid: emergence of a core signaling network. Ann. Rev. Plant Biol. 61, 651-679. doi: 10.1146/annurev-arplant-042809-112122 
Dai, S., Kai, W., Liang, B., Wang, J., Jiang, L., Du, Y., et al. (2018). The functional analysis of SINCED1 in tomato pollen development. Cell Mol. Life Sci. 75, 3457-3472. doi: 10.1007/s00018-018-2809-9

Dietz, K. J., Sauter, A., Wichert, K., Messdaghi, D., and Hartung, W. (2000). Extracellular $\beta$-glucosidase activity in barley involved in the hydrolysis of ABA glucose conjugate in leaves. J. Exp. Bot. 51, 937-944. doi: 10.1093/jxb/51. 346.937

Dong, H., Zhen, Z., Peng, J., Chang, L., Gong, Q., and Wang, N. N. (2011). Loss of ACS7 confers abiotic stress tolerance by modulating ABA sensitivity and accumulation in Arabidopsis. J. Exp. Bot. 62, 4875-4887. doi: 10.1093/jxb/ err 143

Dong, T., and Hwang, I. (2014). Contribution of ABA UDP-glucosyltransferases in coordination of ABA biosynthesis and catabolism for ABA homeostasis. Plant Signal Behav. 9:e28888. doi: 10.4161/psb.28888

Finkelstein, R. (2013). Abscisic Acid synthesis and response. Arabidopsis Book 11:e0166. doi: 10.1199/tab.0166

Fujii, H., Verslues, P. E., and Zhu, J. K. (2011). Arabidopsis decuple mutant reveals the importance of SnRK2 kinases in osmotic stress responses in vivo. Proc. Natl. Acad. Sci. U. S. A. 108, 1717-1722. doi: 10.1073/pnas.1018367108

Furihata, T., Maruyama, K., Fujita, Y., Umezawa, T., Yoshida, R., Shinozaki, K., et al. (2006). Abscisic acid-dependent multisite phosphorylation regulates the activity of a transcription activator AREB1. Proc. Natl. Acad. Sci. U. S. A. 103, 1988-1993. doi: 10.1073/pnas.0505667103

Gonzalez-Guzman, M., Pizzio, G. A., Antoni, R., Vera-Sirera, F., Merilo, E., Bassel, G. W., et al. (2012). Arabidopsis PYR/PYL/RCAR receptors play a major role in quantitative regulation of stomatal aperture and transcriptional response to abscisic acid. Plant Cell 24, 2483-2496. doi: 10.1105/tpc.112.098574

Guan, D., Yang, F., Xia, X., Shi, Y., Yang, S., Cheng, W., et al. (2018). CaHSL1 Acts as a positive regulator of pepper Thermotolerance Under High Humidity and Is Transcriptionally Modulated by CaWRKY40. Front. Plant Sci. 9:1802. doi: 10.3389/fpls.2018.01802

Guang-Cheng, S., Na, L., Zhan-Yu, Z., Shuang-En, Y., and Chang-Ren, C. (2010). Growth, yield and water use efficiency response of greenhouse-grown hot pepper under Time-Space deficit irrigation. Sci. Hortic. 126, 172-179. doi: 10.1016/j.scienta.2010.07.003

Han, Y., Watanabe, S., Shimada, H., and Sakamoto, A. (2019). Leaf endoplasmic reticulum dynamics modulate ss-glucosidase-mediated stress-activated ABA production from its glucosyl ester. J. Exp. Bot. 71, 2058-2071. doi: 10.1093/ jxb/erz528

Han, Y. J., Cho, K. C., Hwang, O. J., Choi, Y. S., Shin, A. Y., Hwang, I., et al. (2012). Overexpression of an Arabidopsis beta-glucosidase gene enhances drought resistance with dwarf phenotype in creeping bentgrass. Plant Cell Rep. 31, 1677-1686. doi: 10.1007/s00299-012-1280-6

Hou, B. Z., Li, C. L., Han, Y. Y., and Shen, Y. Y. (2018). Characterization of the hot pepper (Capsicum frutescens) fruit ripening regulated by ethylene and ABA. BMC Plant Biol. 18:162. doi: 10.1186/s12870-018-1377-3

Iuchi, S., Kobayashi, M., Taji, T., Naramoto, M., Seki, M., Kato, T., et al. (2001). Regulation of drought tolerance by gene manipulation of 9-cis-epoxycarotenoid dioxygenase, a key enzyme in abscisic acid biosynthesis in Arabidopsis. Plant J. 27, 325-333. doi: 10.1046/j.1365-313x.2001.01096.x

Jensen, K., and Moller, B. L. (2010). Plant NADPH-cytochrome P450 oxidoreductases. Phytochemistry 71, 132-141. doi: 10.1016/j.phytochem. 2009.10.017

Ji, K., Kai, W., Zhao, B., Sun, Y., Yuan, B., Dai, S., et al. (2014). SINCED1 and SlCYP707A2: key genes involved in ABA metabolism during tomato fruit ripening. J. Exp. Bot. 65, 5243-5255. doi: 10.1093/jxb/eru288

Jin, J. H., Zhang, H. X., Ali, M., Wei, A. M., Luo, D. X., and Gong, Z. H. (2019). The CaAP2/ERF064 regulates dual functions in pepper: plant cell death and resistance to Phytophthora capsici. Genes (Basel) 10:541. doi: 10.3390/ genes10070541

Kim, A. Y., Kim, H. M., Ma, S. H., Park, S. Y., Dat, M. T., Jang, G., et al. (2019). The promoter of tomato HISTIDINE DECARBOXYLASE A is fruit-specific, and its expression is stably maintained in fruits during ripening. Plant Biotechnol. Rep. 13, 43-50. doi: 10.1007/s11816-018-00512-1

Kim, D. H., Kim, K. H., Kim, D. H., Liu, K. H., Jung, H. C., Pan, J. G., et al. (2008). Generation of human metabolites of 7-ethoxycoumarin by bacterial cytochrome P450 BM3. Drug Metab. Dispos. 36, 2166-2170. doi: 10.1124/dmd. 108.021220
Kim, G.-T., and Tsukaya, H. (2002). Regulation of the biosynthesis of plant hormones by cytochrome P450s. J. Plant Res. 115, 0169-0177.

Kim, H. J., Ok, S. H., Bahn, S. C., Jang, J., Oh, S. A., Park, S. K., et al. (2011). Endoplasmic reticulum- and Golgi-localized phospholipase A2 plays critical roles in Arabidopsis pollen development and germination. Plant Cell 23, 94-110. doi: 10.1105/tpc.110.074799

Kim, H. M., Lee, J. H., Kim, A.-Y., Park, S. H., Ma, S. H., Lee, S., et al. (2016). Heterologous expression of an RNA-binding protein affects flowering time as well as other developmental processes in Solanaceae. Mol. Breed. 36:71.

Krochko, J. E., Abrams, G. D., Loewen, M. K., Abrams, S. R., and Cutler, A. J. (1998). (+)-Abscisic acid 8' -hydroxylase is a cytochrome P450 monooxygenase. Plant Physiol.118, 849-860. doi: 10.1104/pp.118.3.849

Kushiro, T., Okamoto, M., Nakabayashi, K., Yamagishi, K., Kitamura, S., Asami, T., et al. (2004). The Arabidopsis cytochrome P450 CYP707A encodes ABA 8'-hydroxylases: key enzymes in ABA catabolism. EMBO J. 23, 1647-1656. doi: 10.1038/sj.emboj.7600121

Lee, G. Y., Kim, H. M., Ma, S. H., Park, S. H., Joung, Y. H., and Yun, C. H. (2014). Heterologous expression and functional characterization of the NADPHcytochrome P450 reductase from Capsicum annuum. Plant Physiol. Biochem. 82, 116-122. doi: 10.1016/j.plaphy.2014.05.010

Lee, O. R., Nguyen, N. Q., Lee, K. H., Kim, Y. C., and Seo, J. (2017). Cytohistological study of the leaf structures of Panax ginseng Meyer and Panax quinquefolius L. J. Ginseng Res. 41, 463-468. doi: 10.1016/j.jgr.2016.08.001

Lee, S., and Choi, D. (2013). Comparative transcriptome analysis of pepper (Capsicum annuum) revealed common regulons in multiple stress conditions and hormone treatments. Plant Cell Rep. 32, 1351-1359. doi: 10.1007/s00299013-1447-9

Lefebvre, V., North, H., Frey, A., Sotta, B., Seo, M., Okamoto, M., et al. (2006). Functional analysis of Arabidopsis NCED6 and NCED9 genes indicates that ABA synthesized in the endosperm is involved in the induction of seed dormancy. Plant J. 45, 309-319. doi: 10.1111/j.1365-313x.2005. 02622.x

Leng, P., Yuan, B., and Guo, Y. (2014). The role of abscisic acid in fruit ripening and responses to abiotic stress. J. Exp. Bot. 65, 4577-4588. doi: 10.1093/jxb/eru204

Livak, K. J., and Schmittgen, T. D. (2001). Analysis of relative gene expression data using real-time quantitative PCR and the 2(-Delta Delta C(T)) Method. Methods 25, 402-408. doi: 10.1006/meth.2001.1262

Ma, Y., Cao, J., He, J., Chen, Q., Li, X., and Yang, Y. (2018). Molecular mechanism for the regulation of ABA homeostasis during plant development and stress responses. Int. J. Mol. Sci. 19:3643. doi: 10.3390/ijms19113643

Ma, Y., Szostkiewicz, I., Korte, A., Moes, D., Yang, Y., Christmann, A., et al. (2009). Regulators of PP2C phosphatase activity function as abscisic acid sensors. Science 324, 1064-1068.

Mizutani, M. (2012). Impacts of diversification of cytochrome P450 on plant metabolism. Biol. Pharm. Bull. 35, 824-832. doi: 10.1248/bpb.35.824

Nakashima, K., and Yamaguchi-Shinozaki, K. (2013). ABA signaling in stressresponse and seed development. Plant Cell Rep. 32, 959-970. doi: 10.1007/ s00299-013-1418-1

Nambara, E., and Marion-Poll, A. (2005). Abscisic acid biosynthesis and catabolism. Annu. Rev. Plant Biol. 56, 165-185. doi: 10.1146/annurev.arplant. 56.032604 .144046

Nambara, E., Okamoto, M., Tatematsu, K., Yano, R., Seo, M., and Kamiya, Y. (2010). Abscisic acid and the control of seed dormancy and germination. Seed Sci. Res. 20, 55-67. doi: 10.1017/s0960258510000012

Navarro, J., Garrido, C., Flores, P., and Martínez, V. (2010). The effect of salinity on yield and fruit quality of pepper grown in perlite. Span. J. Agric. Res. 8, 142-150. doi: 10.5424/sjar/2010081-1153

Nelson, B. K., Cai, X., and Nebenfuhr, A. (2007). A multicolored set of in vivo organelle markers for co-localization studies in Arabidopsis and other plants. Plant J. 51, 1126-1136. doi: 10.1111/j.1365-313x.2007.03212.x

Nelson, D., and Werck-Reichhart, D. (2011). A P450-centric view of plant evolution. Plant J. 66, 194-211. doi: 10.1111/j.1365-313x.2011.04529.x

Nelson, D. R., Ming, R., Alam, M., and Schuler, M. A. (2008). Comparison of Cytochrome P450 Genes from six plant genomes. Trop. Plant Biol. 1, 216-235. doi: 10.1007/s12042-008-9022-1

Ng, L. M., Melcher, K., Teh, B. T., and Xu, H. E. (2014). Abscisic acid perception and signaling: structural mechanisms and applications. Acta Pharmacol. Sin. 35, 567-584. doi: 10.1038/aps.2014.5 
Nitsch, L. M., Oplaat, C., Feron, R., Ma, Q., Wolters-Arts, M., Hedden, P., et al. (2009). Abscisic acid levels in tomato ovaries are regulated by LeNCED1 and SlCYP707A1. Planta 229, 1335-1346. doi: 10.1007/s00425-009-0913-7

Oh, S. K., Park, J. M., Joung, Y. H., Lee, S., Chung, E., Kim, S. Y., et al. (2005). A plant EPF-type zinc-finger protein, CaPIF1, involved in defence against pathogens. Mol. Plant Pathol. 6, 269-285. doi: 10.1111/j.1364-3703.2005. 00284.x

Okamoto, M., Kuwahara, A., Seo, M., Kushiro, T., Asami, T., Hirai, N., et al. (2006). CYP707A1 and CYP707A2, ertferr Abscisic Acid 8'Hydroxylases, are indispensable for proper control of seed dormancy and germination in Arabidopsis. Plant Physiol. 141, 97-107. doi: 10.1104/pp.106. 079475

Okamoto, M., Tanaka, Y., Abrams, S. R., Kamiya, Y., Seki, M., and Nambara, E. (2009). High humidity induces abscisic acid 8'-hydroxylase in stomata and vasculature to regulate local and systemic abscisic acid responses in Arabidopsis. Plant Physiol. 149, 825-834. doi: 10.1104/pp.108.130823

Omura, T., and Sato, R. (1964). The carbon monoxide-binding pigment of liver microsomes I. Evidence for its hemoprotein nature. J. Biol. Chem. 239, 23702378.

Park, S. Y., Fung, P., Nishimura, N., Jensen, D. R., Fujii, H., Zhao, Y., et al. (2009). Abscisic acid inhibits type $2 \mathrm{C}$ protein phosphatases via the PYR/PYL family of START proteins. Science 324, 1068-1071.

Pathirana, R. (2013). Peppers: Vegetable and Spice Capsicums, 2nd edition, by Paul W. Bosland and Eric J. Votava. N. Z. J. Crop Hortic. Sci. 41, 102-103.

Piffanelli, P., Ross, J. H., and Murphy, D. (1998). Biogenesis and function of the lipidic structures of pollen grains. Sex. Plant Reprod. 11, 65-80. doi: 10.1007/ s004970050122

Priya, R., and Siva, R. (2015). Analysis of phylogenetic and functional diverge in plant nine-cis epoxycarotenoid dioxygenase gene family. J. Plant Res. 128, 519-534. doi: 10.1007/s10265-015-0726-7

Ren, J., Sun, L., Wu, J., Zhao, S., Wang, C., Wang, Y., et al. (2010). Cloning and expression analysis of cDNAs for ABA 8'-hydroxylase during sweet cherry fruit maturation and under stress conditions. J. Plant Physiol. 167, 1486-1493. doi: 10.1016/j.jplph.2010.05.027

Rodrigo, M. J., Alquezar, B., and Zacarias, L. (2006). Cloning and characterization of two 9-cis-epoxycarotenoid dioxygenase genes, differentially regulated during fruit maturation and under stress conditions, from orange (Citrus sinensis L. Osbeck). J. Exp. Bot. 57, 633-643. doi: 10.1093/jxb/erj048

Rodriguez, P. L. (2016). Abscisic Acid Catabolism Generates Phaseic Acid, a molecule able to activate a subset of ABA Receptors. Mol. Plant 9, 1448-1450. doi: 10.1016/j.molp.2016.09.009

Rodríguez-García, M. I., Fernández, M. C., and Alché, J. (1995). Immunocytochemical localization of allergenic protein (Ole e I) in the endoplasmic reticulum of the developing pollen grain of olive (Olea europaea L.). Planta 196, 558-563.

Ruggiero, A., Landi, S., Punzo, P., Possenti, M., Van Oosten, M. J., Costa, A., et al. (2019). Salinity and ABA seed responses in pepper: expression and interaction of ABA core signaling components. Front. Plant Sci. 10:304. doi: 10.3389/fpls. 2019.00304

Sah, S. K., Reddy, K. R., and Li, J. (2016). Abscisic Acid and Abiotic Stress Tolerance in Crop Plants. Front. Plant Sci. 7:571. doi: 10.3389/fpls.2016.00571

Saika, H., Okamoto, M., Miyoshi, K., Kushiro, T., Shinoda, S., Jikumaru, Y., et al. (2007). Ethylene promotes submergence-induced expression of OsABA80x1, a gene that encodes ABA 8'-hydroxylase in rice. Plant Cell Physiol. 48, 287-298. doi: $10.1093 / \mathrm{pcp} / \mathrm{pcm} 003$

Saini, H., and Aspinall, D. (1982). Sterility in wheat (Triticum aestivum L.) induced by water deficit or high temperature: possible mediation by abscisic acid. Funct. Plant Biol. 9, 529-537. doi: 10.1071/pp9820529

Saito, S., Hirai, N., Matsumoto, C., Ohigashi, H., Ohta, D., Sakata, K., et al. (2004). Arabidopsis CYP707As encode (+)-abscisic acid 8'-hydroxylase, a key enzyme in the oxidative catabolism of abscisic acid. Plant Physiol. 134, 1439-1449. doi: 10.1104/pp.103.037614

Schuler, M. A., Duan, H., Bilgin, M., and Ali, S. (2006). Arabidopsis cytochrome P450s through the looking glass: a window on plant biochemistry. Phytochem. Rev. 5, 205-237. doi: 10.1007/s11101-006-9035-z

Schwartz, S. H., Qin, X., and Zeevaart, J. A. (2003). Elucidation of the indirect pathway of abscisic acid biosynthesis by mutants, genes, and enzymes. Plant Physiol. 131, 1591-1601. doi: 10.1104/pp.102.017921
Seiler, C., Harshavardhan, V. T., Rajesh, K., Reddy, P. S., Strickert, M., Rolletschek, H., et al. (2011). ABA biosynthesis and degradation contributing to ABA homeostasis during barley seed development under control and terminal drought-stress conditions. J. Exp. Bot. 62, 2615-2632. doi: 10.1093/jxb/erq446

Seo, M. (2014). "ABA Transmembrane Transport and Transporters," in Abscisic Acid: Metabolism, Transport and Signaling, ed. D.-P. Zhang (Cham: Springer Nature), 47-59. doi: 10.1007/978-94-017-9424-4_3

Seo, M., and Koshiba, T. (2002). Complex regulation of ABA biosynthesis in plants. Trends Plant Sci. 7, 41-48. doi: 10.1016/s1360-1385(01)02187-2

Sharma, S., and Verslues, P. E. (2010). Mechanisms independent of abscisic acid $(\mathrm{ABA})$ or proline feedback have a predominant role in transcriptional regulation of proline metabolism during low water potential and stress recovery. Plant Cell Environ. 33, 1838-1851. doi: 10.1111/j.1365-3040.2010. 02188.x

Simossis, V. A., and Heringa, J. (2005). PRALINE: a multiple sequence alignment toolbox that integrates homology-extended and secondary structure information. Nucleic Acids Res. 33, W289-W294.

Soon, F.-F., Ng, L.-M., Zhou, X. E., West, G. M., Kovach, A., Tan, M. E., et al. (2012). Molecular mimicry regulates ABA signaling by SnRK2 kinases and PP2C phosphatases. Science 335, 85-88. doi: 10.1126/science.1215106

Spaans, S. K., Weusthuis, R. A., Van Der Oost, J., and Kengen, S. W. (2015). NADPH-generating systems in bacteria and archaea. Front. Microbiol. 6:742. doi: $10.3389 /$ fmicb.2015.00742

Steinbrecher, T., and Leubner-Metzger, G. (2017). The biomechanics of seed germination. J. Exp. Bot. 68, 765-783.

Sun, L., Sun, Y., Zhang, M., Wang, L., Ren, J., Cui, M., et al. (2012). Suppression of 9-cis-epoxycarotenoid dioxygenase, which encodes a key enzyme in abscisic acid biosynthesis, alters fruit texture in transgenic tomato. Plant Physiol. 158, 283-298. doi: 10.1104/pp.111.186866

Suttle, J. C., Abrams, S. R., De Stefano-Beltran, L., and Huckle, L. L. (2012). Chemical inhibition of potato ABA- 8 '-hydroxylase activity alters in vitro and in vivo $A B A$ metabolism and endogenous $A B A$ levels but does not affect potato microtuber dormancy duration. J. Exp. Bot. 63, 5717-5725. doi: 10.1093/jxb/ ers146

Tan, B. C., Joseph, L. M., Deng, W. T., Liu, L., Li, Q. B., Cline, K., et al. (2003). Molecular characterization of the Arabidopsis 9-cis epoxycarotenoid dioxygenase gene family. Plant J. 35, 44-56. doi: 10.1046/j.1365-313x.2003. 01786.x

Tan, B. C., Schwartz, S. H., Zeevaart, J. A., and Mccarty, D. R. (1997). Genetic control of abscisic acid biosynthesis in maize. Proc. Natl. Acad. Sci. U. S. A. 94, 12235-12240. doi: 10.1073/pnas.94.22.12235

Tanaka, Y., Nose, T., Jikumaru, Y., and Kamiya, Y. (2013). ABA inhibits entry into stomatal-lineage development in Arabidopsis leaves. Plant J. 74, 448-457. doi: $10.1111 /$ tpj. 12136

Umezawa, T., Sugiyama, N., Mizoguchi, M., Hayashi, S., Myouga, F., YamaguchiShinozaki, K., et al. (2009). Type $2 \mathrm{C}$ protein phosphatases directly regulate abscisic acid-activated protein kinases in Arabidopsis. Proc. Natl. Acad. Sci. U. S. A. 106, 17588-17593. doi: 10.1073/pnas.0907095106

Unlukara, A., Demir, I., Kesmez, D., Éelikkol, T., and Demir, K. (2013). Seed yield and quality of pepper plants grown under salt stress. Afr. J. Biotechnol. 12, 6833-6836.

Verslues, P. E., and Bray, E. A. (2006). Role of abscisic acid (ABA) and Arabidopsis thaliana $\mathrm{ABA}$-insensitive loci in low water potential-induced $\mathrm{ABA}$ and proline accumulation. J. Exp. Bot. 57, 201-212. doi: 10.1093/jxb/erj026

Vishwakarma, K., Upadhyay, N., Kumar, N., Yadav, G., Singh, J., Mishra, R. K., et al. (2017). Abscisic Acid Signaling and abiotic stress tolerance in plants: a review on current knowledge and future prospects. Front. Plant Sci. 8:161. doi: $10.3389 /$ fpls.2017.00161

Vlad, F., Droillard, M. J., Valot, B., Khafif, M., Rodrigues, A., Brault, M., et al. (2010). Phospho-site mapping, genetic and in planta activation studies reveal key aspects of the different phosphorylation mechanisms involved in activation of SnRK2s. Plant J. 63, 778-790. doi: 10.1111/j.1365-313x.2010.04281.x

Voinnet, O., Rivas, S., Mestre, P., and Baulcombe, D. (2003). An enhanced transient expression system in plants based on suppression of gene silencing by the p19 protein of tomato bushy stunt virus. Plant J. 33, 949-956. doi: 10.1046/j.1365313x.2003.01676.x

Wang, Y., Wang, Y., Ji, K., Dai, S., Hu, Y., Sun, L., et al. (2013). The role of abscisic acid in regulating cucumber fruit development and ripening and its 
transcriptional regulation. Plant Physiol. Biochem. 64, 70-79. doi: 10.1016/j. plaphy.2012.12.015

Weng, J. K., Ye, M., Li, B., and Noel, J. P. (2016). Co-evolution of hormone metabolism and signaling networks expands plant adaptive plasticity. Cell 166, 881-893. doi: 10.1016/j.cell.2016.06.027

Xiao, K., Chen, J., He, Q., Wang, Y., Shen, H., and Sun, L. (2020). DNA Methylation Involved in Pepper (Capsicum annuum L.) fruit ripening regulation and interacts with phytohormones. J. Exp. Bot. 71, 1928-1942. doi: 10.1093/jxb/ eraa003

Xu, D., Shi, J., Rautengarten, C., Yang, L., Qian, X., Uzair, M., et al. (2017). Defective Pollen Wall 2 (DPW2) Encodes an Acyl transferase required for rice pollen development. Plant Physiol. 173, 240-255. doi: 10.1104/pp.16.00095

Yang, S. H., and Zeevaart, J. A. (2006). Expression of ABA 8'-hydroxylases in relation to leaf water relations and seed development in bean. Plant J. 47, 675-686. doi: 10.1111/j.1365-313x.2006.02815.x

Yin-Ping, L., Su-Li, S., Chao, Z., Can-Xing, D., and Zhen-Dong, Z. (2019). Cloning and expression analysis of GmCYP82C4 gene in soybean. Chin. J. Oil Crop Sci. 41, 383-390.

Yoshida, T., Obata, T., Feil, R., Lunn, J. E., Fujita, Y., Yamaguchi-Shinozaki, K., et al. (2019). The role of abscisic acid signaling in maintaining the metabolic balance required for Arabidopsis growth under nonstress conditions. Plant Cell 31, 84-105. doi: 10.1105/tpc.18.00766
Zhang, Y., Tan, J., Guo, Z., Lu, S., He, S., Shu, W., et al. (2009). Increased abscisic acid levels in transgenic tobacco over-expressing 9 cis-epoxycarotenoid dioxygenase influence $\mathrm{H} 2 \mathrm{O} 2$ and NO production and antioxidant defences. Plant Cell Environ. 32, 509-519. doi: 10.1111/j.1365-3040.2009.01945.x

Zheng, Y., Huang, Y., Xian, W., Wang, J., and Liao, H. (2012). Identification and expression analysis of the Glycine max CYP707A gene family in response to drought and salt stresses. Ann. Bot. 110, 743-756. doi: 10.1093/aob/mcs133

Zhu, X., Yu, J., Shi, J., Tohge, T., Fernie, A. R., Meir, S., et al. (2017). The polyketide synthase OsPKS2 is essential for pollen exine and Ubisch body patterning in rice. J. Integr. Plant Biol. 59, 612-628. doi: 10.1111/jipb. 12574

Conflict of Interest: The authors declare that the research was conducted in the absence of any commercial or financial relationships that could be construed as a potential conflict of interest.

Copyright (c) 2020 Kim, Park, Ma, Park, Yun, Jang and Joung. This is an open-access article distributed under the terms of the Creative Commons Attribution License (CC BY). The use, distribution or reproduction in other forums is permitted, provided the original author(s) and the copyright owner(s) are credited and that the original publication in this journal is cited, in accordance with accepted academic practice. No use, distribution or reproduction is permitted which does not comply with these terms. 